Please do not remove this page

RMIT

UNIVERSITY

\title{
Diamond as a scaffold for bone growth
}

Fox, Kate; Palamara, Joseph; Judge, Roy; Greentree, Andrew

https://researchrepository.rmit.edu.au/esploro/outputs/9921859144301341/filesAndLinks?institution=61RMIT_INST\&index=null

Fox, K., Palamara, J., Judge, R., \& Greentree, A. (2013). Diamond as a scaffold for bone growth. Journal of Materials Science: Materials in Medicine, 24(4), 849-861. https://doi.org/10.1007/s10856-013-4860-2

Published Version: https://doi.org/10.1007/s10856-013-4860-2

Repository homepage: https://researchrepository.rmit.edu.au

(c) 2013 Springer Science+Business Media New York.

Downloaded On 2023/04/26 22:30:29 +1000 


\section{Abstract:}

Diamond is an attractive material for biomedical implants. In this work, we investigate its capacity as a bone scaffold. It is well established that the bioactivity of a material can be evaluated by examining its capacity to form apatite-like calcium phosphate phases on its surface when exposed to simulated body fluid. Accordingly, polycrystalline diamond (PCD) and ultrananocrystalline diamond (UNCD) deposited by microwave plasma chemical vapour deposition were exposed to simulated body fluid and assessed for apatite growth when compared to the bulk silicon. Scanning electron microscopy and x-ray photoelectron spectroscopy showed that both UNCD and PCD are capable of acting as a bone scaffold. The composition of deposited apatite suggests that UNCD and PCD are suitable for in vivo implantation with UNCD possible favoured in applications where rapid osseointegration is essential.

Keywords: Simulated body fluid, Diamond, Calcium phosphate, Bioactivity

\section{Introduction}

In orthopaedic research, calcium phosphate based implants are important due to the obvious similarities between the natural calcium phosphates in bone and its synthetic varieties. It is thus well established that a metallic implant coated with calcium phosphate, usually synthetic hydroxyapatite, will readily enable bone to bond to the implant. [1-6] Hydroxyapatite has been shown to reduce aseptic loosening in the immediate phase post-implant surgery by enhancing the bone-implant interface by improving bone apposition and osteoconductivity through hydroxyapatite's capacity to form chemical bonds with hard tissue. [1-6] Accordingly, hydroxyapatite has been fabricated as 3D scaffold structures, used as thin-film coating materials, [7-9] as nanoparticles for fracture fillers, [10, 11] and drug delivery vehicles.[12, 13] 
For metallic implants, it has often been considered that implant-bone integration is more mechanical than chemical.[14] Biocompatible metals such as titanium are hindered from integrating with bone by the surface metal oxide rendering the implant inert. Accordingly, it has been suggested that the bone-titanium relationship is a "tight apposition" rather than true metal-bone bonding.[14] Synthetic hydroxyapatite instead has an interfacial response that results in bone bonding.[15] Therefore, coating metallic implants with a hydroxyapatite layer will improve its bonding by providing an adhesion layer between the two surfaces. Studies have shown that the shear strength of hydroxyapatite-coated titanium implants to bone is between 5 to 8 times stronger than an uncoated titanium implant depending on the roughness of titanium and the thickness of the hydroxyaptite. [16-19]

The examination of apatite formation on a substrate in simulated body fluid is known to be useful to predict in vivo bioactivity. [20-23] One method of doing so, was formulated by Kokubo et al [5] who demonstrated that a glass-ceramic, apatite-wollastonite, which was capable of growing a calcium phosphate film in vivo would replicate the same response in vitro if placed in an acellular simulated body fluid with ion concentrations comparable to human blood plasma. Accordingly, this presents a method by which in vivo applicability of a material can, at least partially, be estimated without the need for in vivo experimentation. The technique was further supported by a later study by Kokubo and Takedema [22] who when reviewing the validity of using simulated body fluid to test bioactivity concluded that apatite formation in vitro will be replicated in vivo. The exception to this was third party factors such as contamination in vitro or inflammatory responses in vivo. The link between in vivo and in vitro apatite growth has, however, been at least partially disputed by some research groups [20] who suggest that the in vitro response in simulated body fluid can give both false 
negative as well as false positive results when the sample in placed in vivo. However, it is likely that keeping simulated body fluid ion concentration close to that of human blood plasma will ensure that bioactivity as assessed from acellular simulated body fluid will be predictive of in vivo performance.[20]

Diamond is increasingly becoming interesting as an implantable material.[24-29] Diamondlike coatings and amorphous diamond coatings have been shown to be well osseointegrated with bone.[27, 24] In fact, diamond coated substrates appear to be osseointegrated faster than metallic counterparts particularly in the initial month post-implantation.[24] Chemical vapour deposited (CVD) diamond has been used as a coating material for mandibular plates [26, 25] and as coatings for heart valves[29], joint replacements [30-32] and as a neural simulator[33], whilst fluorescent nanodiamond is being explored for biomarking applications .[34] Diamond is known to possess many favourable qualities for implantation into living tissue including biocompatibility, biostability and for some applications hardness. However, its bioactivity for use as a bone scaffold has not been thoroughly explored. [35, 36] Accordingly, this paper seeks to assess its bioactivity of both ultrananocrystalline diamond (UNCD, grain size $<<1$ micron) and polycrystalline diamond (PCD, grain size 1-5 microns) in hard tissue (bone) using the simulated body fluid bioactivity model. In particular we find that both UNCD and PCD are capable of stimulating apatite deposition onto the substrate. The apatite desposited onto UNCD however appears to be stoichiometrically closer to hydroxyapatite than PCD and thus UNCD may be more bioactive than PCD, that is, possibly better capable of growing bone in vivo. Silicon also showed that it was capable of apatite-like deposition. However, coverage of the apatite-like coating onto the silicon substrate was poor, likely due to its smoothness. Bioactivity is accordingly likely linked to the topographical roughness and surface chemistry of the substrates investigated. 


\section{Materials and Methods}

\subsection{Preparation of samples}

Three sample types were used in this experiment. The substrates for apatite deposition are outlined in Table 1 . Cubes of silicon $\left(5 \times 5 \times 3 \mathrm{~mm}^{3}\right)$ were obtained by dicing a 3mm thick ptype silicon wafer $(<100>)$ with a semi-automatic diamond saw. The cubes were cleaned using standard cleaning methodology of sequential sonication in acetone, methanol and isopropenol and dried under a steady flow of nitrogen gas. Samples were seeded with nanodiamonds by placing silicon substrates into a nanodiamond slurry (3-4 $\mathrm{nm}$ diamond in methanol) and ultrasonicated in the slurry under suspension for 2-3 minutes. Samples were placed in a microwave chemical vapour deposition reactor (iPLAS) to obtain a thin film diamond growth on the top and bottom faces of the cube. The line of sight diamond deposition meant that the sides of silicon cubes were also exposed to the plasma depending on position within the reactor, although such edge effects were unimportant for our study.

PCD samples were prepared by placing seeded silicon into the microwave chemical vapour deposition chamber under a flow of methane and hydrogen (2\% and 98\%, respectively). Similarly, UNCD samples were also prepared by microwave chemical vapour deposition instead under a flow of argon, nitrogen and methane (79\%, $20 \%$ and $1 \%$, respectively). The microwave chemical vapour deposition conditions have been described elsewhere.[33] Samples were exposed to the plasma for 7 hours before samples were rotated and the underside was exposed to the plasma for a further 7 hours.

\subsection{Apatite deposition}


A 1.0 simulated body fluid (SBF) solution with inorganic ion concentrations simulating human blood plasma (Table 2) was prepared following the methodology outlined by Kokubo et al.[5, 22] Briefly, reagent-grade chemicals $\left(\mathrm{NaCl}, \mathrm{NaHCO}_{3}, \mathrm{KCl}, \mathrm{K}_{2} \mathrm{HPO}_{4} \cdot 3 \mathrm{H}{ }_{2} \mathrm{O}\right.$, $\mathrm{MgCl}_{2} \cdot 6 \mathrm{H}_{2} \mathrm{O}, \mathrm{CaCl}_{2}, \mathrm{Na}_{2} \mathrm{SO}_{4}$ ) were dissolved in distilled water. The solution was brought to physiological $\mathrm{pH} 7.35$, using $1 \mathrm{M} \mathrm{HCl}$. The SBF solution was refrigerated at $4^{\circ} \mathrm{C}$ overnight to ensure that no precipitation occurred before experimental use. Prior to use, a glass cover slip was exposed to the solution for 24 hours and precipitation of apatite onto the sample was detected using SEM.[22] No overnight precipitation from the SBF was evident on the sample inner surface of the solution bottle and an assessment of the solution $\mathrm{pH}$ showed negligible change.

Samples outlined in Table 1 were suspended into a polystyrene container with a lid using Vicryl resorbable sutures (Ethicon, Inc). Samples were suspended to ensure apatite deposition onto samples was due to heterogeneous nucleation. The hole in the lid where the sutures exit the polystyrene container was sealed using adhesive tape. The polystyrene container was filled with $11 \mathrm{~mL}$ of SBF solution with respect to the suggested volume defined by the formula $\mathrm{V}_{\mathrm{s}}=\mathrm{S}_{\mathrm{a}} / 10\left(\mathrm{~V}_{\mathrm{s}}\right.$ volume of simulated body fluid $(\mathrm{mL}), \mathrm{S}_{\mathrm{a}}$ the surface area of the sample $\left.\left(\mathrm{mm}^{2}\right)\right)$.[22] Samples were immersed in the SBF solution for periods of 1, 7 and 14 days and kept within an incubator set at $37^{\circ} \mathrm{C}$. SBF solution was not replenished across the 14 day period of experimentation. Post-removal, SBF immersed samples were washed in distilled water and dried under a flow of ultrapure nitrogen gas.

\subsection{Characterisation}

Apatite growth, deposition and desorption was characterised using photography, micrography, roughness calculation and x-ray photoelectron spectroscopy. Using x-ray 
photoelectron spectroscopy we were able to ascertain the chemical composition of samples and estimate apatite growth chemistry.

\subsubsection{Scanning electron microscopy (SEM)}

SEM images were acquired using a JEOL JSM-5910 Scanning electron microscope.

Topographical imaging of samples was performed at an acceleration voltages of $30 \mathrm{kV}$ and imaging of edges was performed at $15 \mathrm{kV}$. Focused ion beam (FIB) milling and SEM image acquisition was attainted using an xT Nova Nanolab 200 FIB/SEM.

\subsubsection{X-ray photoelectron spectroscopy (XPS)}

Analysis of the samples was performed by XPS (Thermo-Fisher K-Alpha), using a Mg Ka radiation source at a power of $300 \mathrm{~W}$. The spot size of analysis was $400 \mathrm{um}$. Sample scanning was performed using the flood gun to compensate for charging and auto height to determine optimal distance between the x-ray beam and the sample. The elements detected in the surface layers (analysis depth 2-5 nm) were observed from the survey spectrum (pass energy $200 \mathrm{eV}$ ) over a range of 0-1100 eV (step size $1 \mathrm{eV}$, dwell time $50 \mathrm{~ms}$ ).

The XPS binding energy (BE) values from high-resolution scans of elemental signals (pass energy $50 \mathrm{eV}$ ) were not charge-corrected with respect to that of adventitious carbon at 284.8 $\mathrm{eV}$ due to scanning being undertaken using the flood gun. In particular, high-resolution XPS spectra were collected for the key elements calcium, carbon, oxygen and phosphorous, identified from the survey spectra. High resolution scanning was undertaken with a step size of $0.1 \mathrm{eV}$ and a dwell time of $50 \mathrm{~ms}$. Data was collected and analysed using Avantage Data System. 


\subsubsection{Surface Roughness}

Roughness was assessed using a XP-2 Stylus Profiler (Ambios Technology, Inc) at a force $0.1 \mathrm{mg}$, scan rate of $0.01 \mathrm{~mm} / \mathrm{s}$ over a distance of $0.5 \mathrm{~mm}$. Each sample was repeated 5 times. Data was analysed using the in-built XP software (Ambios Technology, Inc. 2003).

\section{Results and Discussion}

\subsection{Appearance}

Figure 1 shows a scanning electron micrograph of the diamond growth on the silicon substrates. PCD is shown to have a large number of sheet-like crystals with an average size of between 1 and 5 microns (Fig. 1A). The growth is consistent across the micrograph. The UNCD growth appears to be much rougher with cauliflower morphology typical of UNCD. In the micrograph, individual crystals cannot be seen but each "cauliflower” is approximately 8 - 15 microns (Fig. 1B). It known that UNCD should be a rough surface with diamond crystals in the nanometre range. [33]

Figure 2 shows digital photographs of the sample face after exposure to the simulated body fluid solution for 1, 7 and 14 days (4 separate samples of each type, not the same sample sequentially imaged). It is clear that all three samples appear to change significantly from their initial as-received colour to a more colourful appearance due to thin film interference fringes. Looking first at the silicon samples, it is apparent that after 1 day exposed to SBF, a secondary coating is evident. The coating appears to be patchy and becomes more so as the time period increases. One estimation for the apparent decrease in secondary coverage after 
14 days on the silicon sample is that the secondary coating is unable to adhere to the smooth silicon surface.

Now considering the diamond samples, it is clear that in comparison to the control diamond substrate sample (no SBF exposure), all SBF immersed samples, irrespective of average crystal size, show a change in colour. After 1 day, the UNCD sample looks similar to the untreated UNCD sample, whilst the PCD sample has clearly taken on a blueish shade, particularly in the centre of its exposed face. This suggests that deposition is variable in thickness. After 7 days, the UNCD sample takes on a more yellow façade whilst the PCD sample remains a similar shade to that seen after 1 day. This suggests that in the PCD sample at least, coating thickness may have evened out across the face. The UNCD sample also appears to have a well established and even coating. After 14 days, the reflected colour of both samples dim to become a paler grey (UNCD) or green (PCD). This may signify increases to the HA coating thickness. The optical results provide a good qualitative test, however to quantify the thickness of the HA layer (both as-received and post SBF growth), we employed SEM as discussed below.

The scanning electron microscopy images of the UNCD substrate before and after exposure to SBF are shown in Figure 3. After 1 week, it appears that a uniform growth of an apatitelike coating has precipitated onto the UNCD sample. The growth appears as a thin slate-like film with clear margins between plates. Each plate is between 3-5 microns and the size appears to be uniform across the surface irrespective of the underlying surface roughness of the UNCD. This suggests that apatite-like coating growth is well established and supports the micrograph images. The growth is similar to the film detected by Barrere et al [37]who showed a conformal apatite growth on titanium substrates having 1-5 micron sized globules 
but also found that apatite deposited as a plate-like layer. Here, the growth has covered the naturally occurring peaks or bubbles detected in the original UNCD substrate.

After 2 weeks, the film detected by the micrograph appears, as expected, to be thicker with the margins between plates appearing more like cracks in the film. Cracking of the film is not necessarily undesirable unless the cracks extend to the base of the substrate. It is unknown whether the cracking is a coating effect or has been induced by the post-SBF drying process. Voids in the coating may be a source of bacterial colonisation and thus should be avoided if the coatings were to be used in dental implants.[38] The size of the apatite-like coating plates has increased to approximately 10 microns and growth again appears to the uniform across the surface. Additional nodules of apatite-like growth can also be seen on the surface of the apatite-like coating. These are highly charged by the electron beam. The nodule size is approximately 2 microns and the nodules appear to agglomerate into clusters.

The PCD sample does not appear to initially deposit an apatite-like coating onto its surface. After the first week in SBF, the SEM suggests that little apatite has been deposited on the substrate. It is clear from the micrograph that the inherent PCD crystals are still visible whilst nodules of a highly charged apatite-like coating are sporadically deposited on the surface. After 2 weeks, however, a uniform apatite-like coating is seen across the PCD substrate. The apatite-like coating appears to be a thin film following the contours of the PCD underneath. The layer appears slate-like and similar to that seen on the UNCD samples. Cracking is again evident within the coating with the apatite-like coating plates approximately 10 microns in size. 
The capacity for both UNCD and PCD to act as a substrate for the growth of apatite films appears to contradict the results of Popov et al.[36, 35, 39] that showed that for diamond/amorphous carbon (a-C) films, neither the nanocrystalline diamond (NCD) [35] nor the ultrananocrystalline diamond (UNCD) [39] films were bioactive. Instead, NCD/a-C and UNCD/a-C when exposed to simulated body fluid for 5 and 10 days did not show any apatite layer deposition nor depletion of calcium or phosphorous ions from solution. Accordingly, although it was shown that the films were non-cytotoxic, they were considered bioinert. It is evident from the results of this study that an UNCD and PCD film can effectively "grow" an apatite-like coating. It is clear that both samples have deposited a secondary film.

Similar to the UNCD, after 1 week in simulated body fluid, it is apparent that a thin film apatite-like layer has been deposited onto the silicon sample. The layer, however, is patchy and easily delaminated. As seen on other substrates, the layer appears to be subject to cracking. After 2 weeks, the apatite-like coating deposition appears to still be very thin. Growth is more uniform than that seen at 1 week. The film has no evidence of cracking, however weakened portions of the film can be seen in the micrograph and it is likely that these areas will be the margins subject to subsequent cracking. This area of the silicon substrate showed a constant coat of apatite-like material however other regions as depicted in Fig. 4 shows that this is not the case across the entire substrate. Liu et al [23] performed a similar apatite deposition onto silicon study. In their study, a control $<100>$ silicon wafer was soaked in simulated body fluid for 14 days. It was deemed that a virgin silicon wafer is unable to form apatite on its surface and therefore the substrate is not bioactive. The results of this study, suggest that the silicon is bioactive but rather that the smoothness of the substrate does not allow good conformal growth and can be easily delaminated. 


\subsection{Thickness of coatings}

The thickness of deposited apatite layers were assessed using scanning electron microscopy at high resolution of focussed ion beam milled regions in the centre of the sample faces (Figs. 5-7). After 2 weeks it is evident that both the PCD and UNCD have grown a film of an apatite-like coating on their face. Figure 5 shows the PCD sample. It is clear from the 5B and $\mathrm{C}$ that some variation exists in the film thickness. However, taking an average of the thicknesses across the FIB milled central region and referencing measurements to a reference sample with known thickness, the film was determined to be approximately $410 \mathrm{~nm}$. In comparison, Fig. 6 shows the UNCD sample. The apatite-like layer is considerably thicker than that seen on the PCD substrate. Coating thickness is calculated to be approximately 760 nm. Accordingly, the UNCD appears to grow a layer of apatite-like material twice as thick than that on the PCD. The thickness of growth of apatite-like coating on the UNCD supports the findings of McLeod et al, who noted that on a titanium disc, 1 micron of apatite could be deposited in 21 days using the same simulated body fluid recipe.[40]

The silicon substrate as shown in both Figs 4 and 7 also appears to grow an apatite-like layer after 2 weeks of exposure to the simulated body fluid. It is evident from Fig. 4 that the growth is patchy and non-uniform. Whether the patchy growth occurs due to apatite-like coating deposition or delaminating during handling is not clear at this stage. In any case, the thickness of deposited apatite appears to possibly be over a micron in the larger patches but consistently thinner than this over the majority of the sample face. This may be explained by the area of the substrate analysed for thickness measurements. Looking at the milled central region of the apatite-silicon coating, it is evident that depsotion in this SEM image (Fig. 7A) is conformal and consistent with growth thickness determined to be $250 \mathrm{~nm}$, significantly decreased from the micron deep patches in Fig. 4B. 
The thickness of apatite-like coating deposition was determined to be $410 \mathrm{~nm}$ for PCD, 760 $\mathrm{nm}$ for UNCD and 250-1000 $\mathrm{nm}$ for the silicon substrates. It is clear that UNCD grew a thicker layer of apatite-like coating than PCD. Given the nature of the deposition process, the apatite deposited in the center of the samples will be the thickest area of growth as deposition is reported to be somewhat domed.[40-42] This is likely due to the surface tension between the convex meniscus at the edge of the samples in the SBF solution[42] where within a solution deposition technique such as simulated body fluid the deposition forces are offset.

\subsection{Surface characterisation}

The PCD and UNCD samples exposed to the simulated body fluid also showed elemental evidence of apatite-like phase deposition (Fig. 8). Both samples showed characteristic calcium and phosphorous peaks and a reduction in the carbon signal attributed to the diamond substrate after exposure to SBF. Given the evidence provided by the scanning electron micrographs that the apatite-like layer deposited on the PCD substrate is a thin film, we are able to draw the same conclusion through the XPS results. The carbon signal shown in the survey spectra of the PCD after two weeks in simulated body fluid is still dominant suggesting contribution from the underlying substrate. In the UNCD, where a thicker apatite layer deposited, it is clear that the intensity of the carbon has dropped which suggested minimal if any contribution by the underlying diamond. Instead, carbon is introduced as hydrocarbon $(285 \mathrm{eV})$ and C-O, O-C-O bonds $(286.5 \mathrm{eV})$ rather than as diamond. Figure 9C shows a XPS survey spectra scan of the silicon substrate before and after exposure to simulated body fluid. After 2 weeks, calcium and phosphorous peaks can be detected, however, small silicon peaks can still be detected after exposure. The XPS analysis spot size 
is $400 \mu \mathrm{m}$ and accordingly, it is not surprising that both apatite and silicon can be detected considering the patchy growth seen in the SEM micrographs.

\subsection{Determination of coating type via $\mathrm{Ca} / \mathrm{P}$ ratio}

Hydroxyapatite has stoichiometric calcium to phosphorous ratio $(\mathrm{Ca} / \mathrm{P})$ of 1.67 . Accordingly, the $\mathrm{Ca} / \mathrm{P}$ was calculated for all samples before and after exposure to simulated body fluid. According to Suchanek, the Ca/P ratio for hydroxyapatite can be anywhere between 1.3 and 2.0.[43] $\mathrm{Ca} / \mathrm{P}$ ratios which fall below 1 are not suitable for in vivo implantation as the native acidity of the material as well as its high solubility precludes it.[44] Here, it is evident from Figure 10 that all diamond and silicon samples fell well below the HA stoichiometric ratio of 1.67. This is not uncommon as XPS is a surface sensitive technique and it is well established that calcium depletion occurs on the surface of apatites when compared to the bulk material. $[22,45-49,40]$ The calcium depletion may be due to patchy growth, solution contaminants or influence of the underlying substrate. The UNCD samples were the only samples to fall within the requisite $\mathrm{Ca} / \mathrm{P}$ ratio range of $1.3-2.0$ The average $\mathrm{Ca} / \mathrm{P}$ ratio of these samples was approximately 1.4 and this suggests it may be a calcium phosphate phase somewhere between beta-tricalcium phosphate (stoichiometric $\mathrm{Ca} / \mathrm{P}$ ratio 1.5$)$ and octacalcium phosphate $(\mathrm{Ca} / \mathrm{P}$ 1.3) and possibly dicalcium phosphate ( $\mathrm{Ca} / \mathrm{P} 1.0)$. Having a $\mathrm{Ca} / \mathrm{P}$ around 1.3 is not uncommon since, the formation of octacalcium phosphate is speculated to be a precursor to biologic apatite.[50, 51] A similar $\mathrm{Ca} / \mathrm{P}$ ratio (1.42-1.48) has been attributed to either cation substitution of the $\mathrm{Ca}^{2+}$ ion and/or substitution of $\mathrm{PO}_{4}^{3-}$ with $\mathrm{HPO}_{4}^{2-}$ at the same time utilising calcium vacancies to ensure that the apatite coating remains electrically charge neutral. [52] The $\mathrm{Ca} / \mathrm{P}$ ratio of the PCD substrates however does increase with respect to exposure time and accordingly, as the apatite coating thickness increases, the ratio may increase to within 
Suchanek's ratio,[43] however, it would be possible that the growth is a variety of dicalcium phosphate, a highly dissolvable apatite.

High resolution XPS scans of the apatite exposed substrates showed that each sample had a clear calcium signal after 7 and 14 days. Using the high resolution scan of the calcium signal it is possible to ascertain whether the coating on the substrates was in fact a calcium phosphate material such as hydroxyapatite or a calcium carbonate material. It is known that the $2 p_{3 / 2}$ photoelectron peak of calcium will have a binding energy at $347.5 \mathrm{eV}$ for samples comprising a calcium phosphate.[53] Referring to Table 3, the UNCD, PCD and silicon samples each have a peak indicative of calcium phosphate. Given that the $\mathrm{Ca} / \mathrm{P}$ ratio was below the stoichiometric ratio of traditional hydroxyapatite, it is likely that the coating is a calcium depleted apatite variety but a calcium phosphate nonetheless. In particular, given that both diamond samples have a $\mathrm{Ca} / \mathrm{P}$ ratio close to or over 1.3 , it is highly likely that with more deposition time in SBF biologic apatite will form more closely reflecting hydroxyapatite. [50, 51]

\section{Conclusion}

Apatite films have been successfully deposited on both ultrananocrystalline and polycrystalline diamond substrates and compared with silicon control samples. Diamond and silicon samples exposed to simulated body fluid were able to grow layers thicker than previously detected in the literature suggesting that the bulk diamond substrates were bioactive rather than bioinert as reported by some for composite diamond-amorphous carbon layers.[35, 39] Unexpectedly, silicon appears to be capable of growing an apatite layer though it appears that its inherent low surface roughness makes it difficult for apatite coatings 
to adhere long term. The capacity of diamond to grow apatite may be related to the nanoroughness of the substrates enabling apatite to nucleate within crevices. It however appears that the apatite layer was not hydroxyapatite but rather calcium depleted apatite, possibly octacalcium phosphate, a dissolvable precursor to hydroxyapatite. In any case, the study suggests that both ultrananocrystalline and polycrystalline diamond are suitable for in vivo implantation with a possible preference towards ultrananocrystalline diamond where fast osseointegration is required.

Acknowledgements

A.D.G. acknowledges the Australian Research Council for financial support (Project No. DP0880466). This work was supported by the University of Melbourne Interdisciplinary Seed Funding scheme. K.F. is financially supported by the Australian Research Council (ARC) through its Special Research Initiative (SRI) in Bionic Vision Science and Technology grant to Bionic Vision Australia (BVA) and by the University of Melbourne Research Collaboration Grant scheme. K.F acknowledges the support of Surgical Design and Manufacture Ltd and Prof. Steven Prawer. The authors wish to acknowledge the facilities, and the scientific and technical assistance, of the Australian Microscopy \& Microanalysis Research Facility at the RMIT Microscopy \& Microanalysis Facility, at RMIT University and Dr Jiri Cervenka for FIB-SEM assistance.

\section{References}

1. Baker K, Anderson M, Oehlke S, Astashkina A, Haikio D, Drelich J et al. Growth, characterization and biocompatibility of bone-like calcium phosphate layers biomimetically deposited on metallic substratate. Materials Science \& Engineering C. 2006;26(8):1351-60. 2. Stigter M, Bezemer J, de Groot K, Layrolle P. Incorporation of different antibiotics into carbonated hydroxyapatite coatings on titanium implants, release and antibiotic efficacy. Journal of Controlled Release. 2004;99(1):127-37. doi:10.1016/j.jconrel.2004.06.011. 3. Choi JM, Kim HE, Lee IS. Ion-beam-assisted deposition (IBAD) of hydroxyapatite coating layer on Ti-based metal substrate. Biomaterials. 2000 21(5):469-73. 
4. Knabe C, Berger G, Gildenhaar R, Klar F, Zreiqat H. The modulation of osteogenesis in vitro by calcium titanium phosphate coatings Biomaterials. 2004;25(20):4911-9. .

5. Kokubo T, Kushitani H, Sakka S, Kitsugi T, Yamamuro T. Solutions able to reproduce in vivo surface-structure changes in bioactive glass-ceramic A-W. Journal of Biomedical Materials Research. 1990;24(6):721-34.

6. Coathup M, Blackburn J, Goodship A, Cunningham J, Smith T, Blunn G. Role of hydroxyapatite coating in resisting wear particle migration and osteolysis around acetabular components. Biomaterials. 2005;26(19):4161-9.

7. Sun LM, Berndt CC, Gross KA, Kucuk A. Material fundamentals and clinical performance of plasma-sprayed hydroxyapatite coatings: A review. Journal of Biomedical Materials

Research. 2001;58(5):570-92. doi:10.1002/jbm.1056.

8. Sun LM, Berndt CC, Khor KA, Cheang HN, Gross KA. Surface characteristics and dissolution behavior of plasma-sprayed hydroxyapatite coating. Journal of Biomedical Materials Research. 2002;62(2):228-36. doi:10.1002/jbm.10315.

9. Cheang P, Khor KA. Addressing processing problems associated with plasma spraying of hydroxyapatite coatings. Biomaterials. 1996;17(5):537-44. doi:10.1016/0142-

9612(96)82729-3.

10. Ginebra MP, Traykova T, Planell JA. Calcium phosphate cements as bone drug delivery systems: A review. Journal of Controlled Release. 2006;113(2):102-10.

doi:10.1016/j.jconrel.2006.04.007.

11. Tadic D, Epple M. A thorough physicochemical characterisation of 14 calcium phosphate-based bone substitution materials in comparison with natural bone. Biomaterials 2004;25(6):987-94.

12. Venkatesan P, Puvvada N, Dash R, Kumar BNP, Sarkar D, Azab B et al. The potential of celecoxib-loaded hydroxyapatite-chitosan nanocomposite for the treatment of colon cancer. Biomaterials. 2011;32(15):3794-806. doi:10.1016/j.biomaterials.2011.01.027.

13. Uskoković V, Uskoković D. Nanosized hydroxyapatite and other calcium phosphates: Chemistry of formation and application as drug and gene delivery agents. Journal of Biomedical Materials Research Part B: Applied Biomaterials. 2011;96B:152-91.

14. Nath S, Basu B. Materials for orthopedic applications. In: Basu B, Katti D, Kumar A, editors. Advanced Biomaterials: Fundamentals, processing and applications. Hoboken, New Jersey.: John Wiley and Sons. 2009

15. Klein C, Groot Kd. Implant systems based on bioactive ceramics. In: Heimke G, editor. Osseo-integrated implants: Implants in oral and ENT surgery. Boca Raton: CRC Press; 1990. p. 193-208.

16. Yoshinari M, Klinge B, Derand T. The biocompatibility (cell culture and histologic study) of hydroxyl-apatite-coated implants created by ion beam dynamic mixing. Clinical Oral Implants Research. 1996; 7:96-100.

17. Cook S, Thomas K, Brinker M. Bioactive ceramic coatings for orthopaedic and dental implant applications. Blood compatible materials and devices: Perspectives towards the 21st century. Lancaster: Technomic publishing company; 1991.

18. Coathup MJ, Blunn GW, Flynn N, Williams C, Thomas NP. A comparison of bone remodelling around hydroxyapatite-coated, porous-coated and grit-blasted hip replacements retrieved at post-mortem. Journal of Bone and Joint Surgery-British Volume.

2001;83B(1):118-23. doi:10.1302/0301-620x.83b1.10062.

19. Sousa SR, Barbosa MA. Effect of hydroxyapatite thickness on metal ion release from

Ti6Al4V substrates. Biomaterials. 1996;17(4):397-404. doi:10.1016/0142-9612(96)89655-4. 20. Bohner M, Lemaitre J. Can bioactivity be tested in vitro with SBF solution Biomaterials. 2009;30 2175-9. 
21. Combes C, Rey C. Adsorption of proteins and calcium phosphate materials bioactivity. Biomaterials. 2002;23:2817-23.

22. Kokubo T, Takadama H. How useful is SBF in predicting in vivo bone bioactivity? Biomaterials. 2006;27:2907-15.

23. Liu X, Fu RKY, Poon RWY, Chen P, Chu PK, Ding C. Biomimetic growth of apatite on hydrogen-implanted silicon. Biomaterials. 2004;25(25):5575-81.

doi:10.1016/j.biomaterials.2004.01.015.

24. Jaatinen J, Korhonen R, Pelttari A, Helminen H, Korhonen H, Lappalainen R et al. Early bone growth on the surface of titanium implants in rat femur is enhanced by an amorphous diamond coating. Acta Orthop. 2011;82(4):499-503.

25. Papo MJ, Catledge SA, Vohra YK. Mechanical wear behavior of nanocrystalline and multilayered diamond coatings on temporomandibular joint implants. J Mat Sci-Mat In Medicine. 2004;15:773.

26. Fries M, Vohra Y. Nanostructured diamond film deposition on curved surfaces of metallic temporomandibular joint implant. J Phys D: Appl Phys 2002;35(20 ):L105-L7. 27. Guglielmotti MB, Renou S, Cabrini RL. A histomorphometric study of tissue interface by laminar implant test in rats. Int J Oral Maxillofac Implants. 1999 14(4):565-70.

28. Booth L, Catledge S, Nolen D, Thompson R, Vohra Y. Synthesis and Characterization of Multilayered Diamond Coatings for Biomedical Implants. Materials. 2011; 4:857-68.

29. Jozwik K, Karczemska A. The new generation Ti6Al4V artificial heart valve with nanocrystalline diamond coating on the ring and with Derlin disc after long-term mechanical fatigue examination. Diam Relat Mater. 2007;16:1004.

30. Aspenberg P, Anttila A, Konttinen YT, Lappalainen R, Goodman SB, Nordsletten L et al. Benign response to particles of diamond and SiC: bone chamber studies of new joint replacement coating materials in rabbits. Biomaterials. 1996;17(8):807-12. doi:10.1016/01429612(96)81418-9.

31. Mattei L, Di Puccio F, Piccigallo B, Ciulli E. Lubrication and wear modelling of artificial hip joints: A review. Tribology International. 2011;44(5):532-49.

doi:10.1016/j.triboint.2010.06.010.

32. Saikko V, Ahlroos T, Calonius O, Keränen J. Wear simulation of total hip prostheses with polyethylene against CoCr, alumina and diamond-like carbon. Biomaterials.

2001;22(12):1507-14. doi:10.1016/s0142-9612(00)00306-9.

33. Garrett DJ, Ganesan K, Stacey A, Fox K, Meffin H, Prawer S. Ultra-nanocrystalline diamond electrodes: Optimisation for neural Stimulation. J Neural Eng. 2012;9(1):10.

34. Aharonovich I, Castelletto S, Simpson D A, Su C-H, Greentree A D, S P. Diamond-based single-photon emitters. Rep Prog Phys. 2011;74(7):076501.

35. Popov C, Kulisch W, Jelinek M, Bock A, Strnad J. Nanocrystalline diamond/amorphous carbon composite films for applications in tribology, optics and biomedicine. Thin Solid Films. 2006;494(1-2):92-7. doi:10.1016/j.tsf.2005.07.163.

36. Popov C, Kulisch W, Reithmaier JP, Dostalova T, Jelinek M, Anspach N et al.

Bioproperties of nanocrystalline diamond/amorphous carbon composite films. Diamond and Related Materials. 2007;16(4-7):735-9. doi:10.1016/j.diamond.2006.12.001.

37. Barrere F, van Blitterswijk CA, de Groot K, Layrolle P. Influence of ionic strength and carbonate on the Ca-P coating formation from $\mathrm{SBF} \times 5$ solution. Biomaterials.

2002;23(9):1921-30. doi:10.1016/s0142-9612(01)00318-0.

38. Faig-Martia J, Gil-Murb FJ Hydroxyapatite coatings in prosthetic joints. Rev Esp Cir Ortop Traumatol 2008;52 113-20.

39. Kulisch W, Popov C, Gilliland D, Ceccone G, Reithmaier JP, Rossi F. UNCD/a-C nanocomposite films for biotechnological applications. Surface and Coatings Technology. 2011;206(4):667-75. doi:10.1016/j.surfcoat.2011.03.057. 
40. McLeod K, Kumar S, Dutta NK, Smart RS, Voelcker NH, Anderson GI. X-ray photoelectron spectroscopy study of the growth kinetics of biomimetically grown hydroxyapatite thin-film coatings. Applied Surface Science. 2010;256(23):7178-85. doi:10.1016/j.apsusc.2010.05.047.

41. Chosa N, Taira M, Saitoh S, Sato N, Araki Y. Characterization of apatite formed on alkaline-heat-treated Ti. Journal of Dental Research. 2004;83(6):465-9.

42. Ben-Nissan B, Chai CS, Gross KA. Effect of solution ageing on sol-gel hydroxyapatite coatings. Bioceramics, Vol 10. 1997.

43. Suchanek W, Yoshimura M. Processing and properties of hydroxyapatite-based biomaterials for use as hard tissue replacement implants. Journal of Materials Research. 1998;13(1):94-117.

44. Dorozhkin SV. Bioceramics of calcium orthophosphates. Biomaterials. 2010;31:1465-85. 45. Kokubo T, Kim H-M, Kawashita M. Novel bioactive materials with different mechanical properties Biomaterials. 2003;24:2161-75.

46. Kokubo T, Ito S, Shigematsu M, Sakka S, Yamamuro T JMS-. Fatigue and lifetime of bioactive glass-ceramic A-W containing apatite and wollastonite. Journal of Materials Science. 1987;22:4067-70.

47. Lu X, Leng Y. Theoretical analysis of calcium phosphate precipitation in simulated body fluid. Biomaterials. 2005;26(10):1097-108. doi:10.1016/j.biomaterials.2004.05.034. 48. Peng P, Kumar S, Voelcker NH, Szili E, Smart R St.C, Griesser H. Thin calcium phosphate coatings on titanium by electrochemical deposition in modified simulated body fluid. Journal of Biomedical Materials Research. 2006;76A(2):347-55.

49. Tanahashi M \& Matsuda T 1997. Surface functional group dependence on apatite formation on self-assembled monolayers in a simulated body fluid. Journal of Biomedical Materials Research. 1997;34(3):305-15.

50. LeGeros RZ. Fundamentals of hydroxyapatite and related calcium phosphates In: Basu B, Katti D, Kumar A, editors. Advanced Biomaterials: Fundamentals, processing and applications. Hoboken, New Jersey. John Wiley and Sons, ; 2009

51. Brown WE, Smith JP, Frazier AW, Lehr JR. Crystallographic and chemical relations between octacalcium phosphate and hydroxyapatite. Nature. 1962;196(4859):1050-\&. doi:10.1038/1961050a0.

52. Chou Y-F, Chiou W-A, Xu Y, Dunn JCY, Wu BM. The effect of pH on the structural evolution of accelerated biomimetic apatite. Biomaterials. 2004;25(22):5323-31. doi:10.1016/j.biomaterials.2003.12.037.

53. Amin M, Randeniya L, Bendavid A, Martin P, E. P. Amorphous carbonated apatite formation on diamond-like carbon containing titanium oxide. Diamond and Related Materials. 2009;18:1139-44. 
Table 1. Details of sample preparation and the abbreviations used for each sample, $n=4$

\begin{tabular}{|l|l|l|}
\hline Sample & Method & Abbreviation \\
\hline Silicon cube & As received & $\mathrm{Si}$ \\
\hline Polycrystalline diamond & CVD layer on silicon & PCD \\
\hline Ultrananocrystalline diamond & CVD layer on silicon & UNCD \\
\hline
\end{tabular}


Table 2: Concentration of ions in SBF as compared to that in human blood plasma

\begin{tabular}{|l|l|l|}
\hline Ion Concentration & Human Blood Plasma (mM) & Simulated Body Fluid (mM) \\
\hline $\mathrm{Na}^{+}$ & 142.0 & 142.0 \\
\hline $\mathrm{K}^{+}$ & 5.0 & 5.0 \\
\hline $\mathrm{Mg}^{2+}$ & 1.5 & 1.5 \\
\hline $\mathrm{Ca}^{2+}$ & 2.5 & 2.5 \\
\hline $\mathrm{Cl}^{-}$ & 103.0 & 147.8 \\
\hline $\mathrm{HCO}^{3-}$ & 27.0 & 4.2 \\
\hline $\mathrm{HPO}^{2-}{ }_{4}$ & 1.0 & 1.0 \\
\hline $\mathrm{SO}^{2-}{ }_{4}$ & 0.5 & 0.5 \\
\hline
\end{tabular}


Table 3: Binding energy of the Ca2p signal as determined by high resolution XPS scanning. The binding energy of the peaks was ascertained using Avantage Data System curve fitting software.

\begin{tabular}{|c|l|l|l|}
\hline \multirow{2}{*}{ Sample } & \multicolumn{2}{|c|}{ Time } & \multicolumn{2}{c|}{ Binding energy (eV) } \\
\cline { 3 - 4 } & & Ca $2 \mathrm{p}_{3 / 2}$ & Ca2 $\mathrm{p}_{1 / 2}$ \\
\hline \multirow{2}{*}{ PCD } & 7 days & 347.5 & 351.2 \\
\cline { 2 - 4 } & 14 days & 347.48 & 350.88 \\
\hline UNCD & 7 days & 347.58 & 351.28 \\
\cline { 2 - 4 } & 14 days & 347.58 & 351.98 \\
\hline Silicon & 7 days & 347.88 & 351.38 \\
\cline { 2 - 4 } & 14 days & 347.58 & \\
\hline
\end{tabular}


Figure 1: Diamond growth on the silicon cube; (A) PCD with average grain size 1-5 microns and (B) UNCD with average grain size of order a few nanometres. Note that the 'bubbles' that can be seen in the UNCD film are not the nanodiamond grains, but instead show variability in the grown film. Growth conditions for the samples are found in section 2.1 .

Figure 2: Photographic representation of samples exposed to simulated body fluid for 0, 1, 7 and 14 days using an Olympus digital camera. The silicon sample appears to develop a secondary coating within 1 day however, the coating appears to delaminate over time. Both diamond samples, UNCD and PCD appear to also develop a secondary coating after 1 day as represented by a change in the colour of the samples. The UNCD shows a more consistent coating than the PCD. Each square above represents a $5 \mathrm{~mm} \times 5 \mathrm{~mm}$ sample area.

Figure 3: Scanning electron micrographs of the samples exposed to simulated body fluid for 0, 7 and 14 days (9 samples). Each micrograph displays a new sample exposed to the simulated body fluid for the specified time period rather than a sequentital analysis of each substrate. It is clear that apatite desposition on the diamond and silcicon substrates has occurred and is conformal ,and that after 2 weeks, the UNCD, PCD and silicon substrates are completely covered. The scale bar represents 10 microns. Beneath the scanning electron micrographs is a graph showing the changing roughness of the samples over time. It is clear that deposition of apatite increases sample roughness with maximum roughness achieved after 1 week. Both diamond samples have a average roughness between 200-400 nm with the UNCD having an increased roughness indicating that the stylus can distinguish the nano features of the UNCD. Silicon has a low average roughness. Apatite deposition appears to follow the same roughness cycle across all samples whereby roughness will increase for week 1 and then begin to decrease as the apatite deposition thickens and conforms to the underlying substrate. The error bars indicate the variability observed from 5 stylus positions across a single sample.

Figure 4: (A) SEM image of the thickness of the apatite layer deposited on silicon after 2 weeks in SBF showing the patchy nature of apatite-like coating deposition on silicon. Apatite-like coating growth appears to be clustered. (B) shows a high resolution image of the same coating.

Figure 5: A SEM image of the PCD sample after 2 weeks in SBF. (A) shows the rough surface topography of the apatite coating; (B) shows this same coating under a 52 degree tilt showing variation in peak height; (C) shows the FIB milled section in the apatite sample; and (D) displays the interface between the apatite and the underlying diamond. Using the image in (D) it is possible to estimate of coating thickness to be $407.5 \mathrm{~nm}$.

Figure 6: A SEM image of the UNCD sample after 2 weeks in SBF. (A) shows the rough surface topography of the apatite coating; (B) shows this same coating under a 52 degree tilt showing variation in peak height; (C) shows the FIB milled section in the apatite sample; and (D) displays the interface between the apatite and the underlying diamond. Using the image in (D) it is possible to estimate of coating thickness to be $760 \mathrm{~nm}$.

Figure 7: A SEM image of the silicon sample after 2 weeks in SBF. (A) shows the surface topography of the apatite coating; (B) shows this same coating under a 52 degree tilt showing consistency in peak height and the beginning of the milling process; (C) shows the FIB milled section in the apatite sample; and (D) displays the interface between the apatite and the underlying silicon. Using the image in (D) it is possible to estimate of coating thickness to be $250 \mathrm{~nm}$.

Figure 8: XPS survey spectra of (A) PCD;(B) UNCD and (C) Silicon before and after exposure to simulated body fluid for 2 weeks. Calcium and Phosphorous can be detected on all three substrates after exposure to SBF suggesting apatite deposition. The X-axis corresponds to the binding energy $(\mathrm{eV})$ and the $\mathrm{Y}$-axis is in arbitrary units

Figure 9: The calcium to phosphorous ratio of substrates as determined from elemental analysis of XPS survey spectra at the appropriate time periods. UNCD appears to be the most bioactive scaffold for implantation as it more closely represents the more desirable apatite varieties for in vivo sample implantation. 